\title{
Acknowledgment of Guest Editors
}

Dr. Benjamin and his editorial team acknowledge the generous and valuable assistance from the following scientists who served as Guest Editors for manuscripts considered for publication in Volume 45 of the Journal of Experimental Psychology: Learning, Memory, and Cognition.

$\begin{array}{llll}\begin{array}{l}\text { M. Teresa Bajo } \\ \text { Marc Brysbaert }\end{array} & \text { Wendy S. Francis } & \text { Michael Inzlicht } & \begin{array}{l}\text { John Paul Minda } \\ \text { Jason Moser }\end{array} \\ \text { Laura Carlson } & \text { David A. Gallo } & \begin{array}{l}\text { Colleen Kelley } \\ \text { Sachiko Kinoshita }\end{array} & \text { John Philbeck } \\ \begin{array}{l}\text { Michael D. Dodd } \\ \text { John Dunlosky }\end{array} & \text { William S. Horton } & \text { Robert H. Logie } & \text { Jeffrey Starns }\end{array}$

\section{Acknowledgment of Ad Hoc Reviewers}

Dr. Benjamin and his editorial team acknowledge the generous and valuable help from the following scientists who, in addition to the Editorial Board members, reviewed manuscripts submitted for publication in Volume 45 of the Journal of Experimental Psychology: Learning, Memory, and Cognition.

Fabian Ache Rakefet Ackerman

Frans Adriaans

Ada Aka

Melisa Akan

Aleksandar Aksentijevic

F.-Xavier Alario

Tuninetti Alba

Hamad S. Aldosari

Daniel Algom

Amit A. Almor

Santiago Alonso-Díaz

Lyneé Alves*

Andrei Amatuni*

Brian A. Anderson

Francis Truitt Anderson

Nicola Anderson

Bernhard Angele

Ulrich Ansorge

Inés Antón-Méndez

Kim Archambeau

Robert Ariel

Blair Armstrong

Jennifer Arnold

Ivan K. Ash

Nathaniel Ashby

Jane Ashby

Nart Atalay

Nina Attridge

Maria Augustinova

Frederik Aust

Joe Austerweil

Serine Avetisyan*

Edward Awh

Peter Ayton

Martijn Baart

Giyeul Bae

Heather Bailey

M. Teresa Bajo

Fuat Balci

Hunter Ball
Tyler Bancroft

Alixandra Barasch

Anthony Barnhart

Amanda J. Barnier

Arthur Baroody

Hilary Barth

Laura Batterink

Ute J. Bayen

Paul Bays

Philip Beaman

Maria Soledad Beato

Anne Lauren Beatty-Martinez

Christos Bechlivanidis

Melissa Renee Beck

Sarah R. Beck

Mark W. Becker

Tom Beckers

Barend Beekhuizen

Tom Beesley

Raoul Bell

Andrea Bender

Roland Benoit

Gershon Ben-Shakhar

Tessa Bent

Lynne Bernstein

Dorthe Berntsen

Miri Besken

Matt Bezdek

Michał Białek

James Bisby

Jovana Bjekic

Robert A. Bjork

Mark R. Blair

Hazel I. Blythe

Susan Bobb

Tres Bodet*

Annika Boldt

Charlotte Bonardi

Patrick Bonin

Sarah Bookbinder

Arielle Borovsky

Mona Roxana Botezatu
Luc Bovens

Holly Bowen

Ty Boyer

Elisabeth Bradford

Senne Braem

David William Braithwaite

Neil Robert Bramley

Holly P. Branigan

Elizabeth Brannon

Mara Breen

Richard Breheny

Laurel Brehm

Neil Brewer

David Bridgett

Victoria Bridgland*

Anne Britt

Arndt Bröder

Trevor Brothers

Alan S. Brown

Gordon Brown

Norman R. Brown

Sarah A. Brown

John Bulevich

Federica Bulgarelli

Nyssa Bulkes

Christian Burgers

Alexander Burgoyne

Jennifer S. Burt

Daniel Bush

Andres Buxo-Lugo

Ruth M. J. Byrne

Cristina Cacciari

Matthew Cain

Catherine Caldwell-Harris

Dustin P. Calvillo

Valérie Camos

Guillermo Campoy

Paolo Canal

Jeremy B. Caplan

Valerio Capraro

Curt Carlson
Matthew Carlson

Alexis Catherine Carpenter

Gareth Carrol

Paulo Carvalho

Naomi Caselli

Giovanni Cassani

Alan Castel

Kyle R. Cave

Craig Chambers

Emmanuel Chemla

Jenn-Yeu Chen

Yiya Chen

Fabienne Chetail

Kimberly Chiew

Jane Childers

Seth Chin-Parker

Yu-Chin Chiu

Yang Seok Cho

Wonil Choi

Shoham Choshen-Hillel

Wing Yee Chow

Liz Chrastil

Kiel Christianson

Evangelia Chrysikou

Elizabeth Chua

Steven E. Clark

Hannah Claussenius-Kalman*

Alexandra A. Cleland

Catherine A. Clement

Pedro L. Cobos

Dale J. Cohen

Emily Rebecca Cohen-Shikora

Sarah Colby

Lorenza S. Colzato

Markus Conci

Kathy Conklin

Louise Connell

Cynthia M. Connine

Erin Conwell

Anne E. Cook

John Cook

Martin Corley 
Ruth Elizabeth Corps

Michael Cortese

Milvia Cottini

Nelson Cowan

Fergus I. M. Craik

Amy H. Criss

Matt Crossley

Nicole Cruz

Chris Cummins

Tim Curran

Anne Cutler

Andree-Ann Cyr

Junyi Dai

Xianchi Dai

Rick Dale

Eddy J. Davelaar

Lisa Davidson

Catherine Davies

Colin J. Davis

Sara Davis

Matt Davis

Tyler Davis

Felipe De Brigard

Angela de Bruin

Simon De Deyne

Vincent de Gardelle

Jan De Houwer

Wim De Neys

Marci S. DeCaro

Tamar Degani

Michael DeKay

Fabio Del Missier

Andrew R. Delamater

Chiara Della Libera

Jerker Denrell

Kobe Desender

Theresa Desrochers

Melissa DeWolf

Mandeep Dhami

Chris Dickinson

David Dignath

Laura Dilley

Brian Dillon

Xianfeng Ding

Nicolas Dirix

Peter Dixon

Ian G. Dobbins

Francesco Donato*

Igor Douven

W. Jay Dowling

Gabriel Doyle

Marko Dragojevic

Jan Drugowitsch

Audrey Duarte

Chad Dube

Sarah DuBrow

Nicolas Dumay

Katherine Duncan

Simon Durrant

Veena Dhar Dwivedi

Alexandra S. Dylman

Kathleen M. Eberhard
Ullrich K. H. Ecker

Maria Eckstein

Miguel Eckstein

Pierce Edmiston

Tobias Egner

Gilles O. Einstein

Shawn Ell

Emily M. Elliott

Paul Edward Engelhardt

Christopher Erb

Edgar Erdfelder

Ido Erev

Thorsten Michael Erle

Alexandra Ernst

Myrthe Faber

Xiaoping Fang

George Farmer

Thomas A. Farmer

Simon Farrell

Jonathan M. Fawcett

Michael Fender

Kimberly Fenn

Eunice G. Fernandes

Mario Ferreira

Victor Ferreira

Joshua Fiechter

Klaus Fiedler

Susann Fiedler

Mario Fific

Ruth Filik

Martin H. Fischer

Simon Fischer-Baum

Stanka A. Fitneva

Joseph Fitzgerald

Heather Flowe

Jocelyn R. Folk

Rebecca Foote

Francesca Foppolo

Stephani Foraker

Kenneth I. Forster

Alice Foucart

Daryl Fougnie

Tom Foulsham

Katherine Fox-Glassman

Wendy S. Francis

Michael Frank

Stella Frank

Scott Fraundorf

Max Freeman

Ori Friedman

Steven Frisson

Rebecca L. A. Frost

Miriam Gade

Christina L. Gagné

Alexia Galati

Niall Galbraith

Chiara Gambi

Matthias Gamer

Eyal Gamliel

Xuefei Gao

Zaifeng Gao

Oscar Garcia-Leal
Alan Garnham

Nicholas Gaspelin

Carolina Gattei

Bertram Gawronski

Surya Gayet

Karl R. Gegenfurtner

Joy Geng

Dedre Gentner

Sebastian Geukes

Thomas Geyer

Edward Gibson

Sam Gilbert

Adrian Gilmore

Arthur Glenberg

Andreas Glöckner

Aline Godfroid

Adele Goldberg

Deborah Goldfarb

Stephen D. Goldinger

Matthew Goldrick

Micah Goldwater

Tianwei Gong*

Corentin Gonthier

Cleotilde Gonzalez

Peter C. Gordon

Simon Gorin

David Gow

Jonathan Grainger

DeMond Grant

David W. Green

Melanie Green

Joshua Greene

Tobias Greitemeyer

Andrea Greve

Oren Griffiths

Christian Grillon

Tricia Guerrero

Jason William Gullifer

Thomas Gunter

Todd M. Gureckis

Eva Gutierrez

Julia Haaf

Wibke Maria Hachmann

Constantinos Hadjichristidis

York Hagmayer

Hilde Haider

Vered Halamish*

Georg Halbeisen

Geoffrey Hall

Kelsi Hall*

Lars Hall

Karina Hamamouche

Zach Hambrick

Maciej Hanczakowski

Simon J. Handley

Adam J. L. Harris

Robert Hartsuiker

Olaf Hauk

Sayuri Hayakawa

Lisheng $\mathrm{He}$

Karl Healey

Daniel Heck

Craig Hedge
Mary Hegarty

Sebastien Helie

Chelsea Helion

Daphna Heller

Pernille Hemmer

Peter Hendrix

Linda A. Henkel

Arturo E. Hernandez

Hal Hershfield

Paula Hertel

Stefan M. Herzog

Clayton Hickey

Jason L. Hicks

Philip A. Higham

Thomas Hills

Florian Hintz

Douglas Hintzman

Patricia Hirsch

William E. Hockley

Paul Hoffman

Janina Anna Hoffmann

Geoff Hollis

Linus Holm

Kevin Holmes

Bernhard Hommel

George Houghton

Joseph Woodworth Houpt

Liv Hoversten

Kuan-Jung Huang

Timothy L. Hubbard

David E. Huber

Amanda Huensch

Markus Huff

Mark J. Huff

Robert Wyn Hughes

John E. Hummel

Almut Hupbach

Mark John Hurlstone

Edward Matthew Husband

Thomas Hutcheon

Jukka Hyona

Albrecht Inhoff

Aine Ito

Iva Ivanova

Carrie Jackson

Cassandra Jacobs

Allison Jaeger

Lena Jaeger

Ariel James

Lori James

Taylor James*

Markus Janczyk

Steve M. J. Janssen

Debra Jared

Andrew F. Jarosz

Chris Jarrold

Juhani Järvikivi

Alexandra Jesse

Jiefeng Jiang

Yuhong V. Jiang

William Jimenez Leal

Brendan Johns 
Jeffrey Johnson

Samuel G. B. Johnson

Dylan M. Jones

Lara L. Jones

Matt Jones

Winston Jones

Olessia Jouravlev

Barbara J. Juhasz

Saiki Jun

Peter Juslin

Ion Juvina

Johanna K. Kaakinen

Edith Kaan

Michael Kahana

Daniel Kaiser

Elsi Kaiser

Michael Kalish

Sean H. K. Kang

Natalia Karelaia

Hossein Karimi

Chris Karvetski

Michael P. Kaschak

Florian Kattner

Tobias Katus

Juergen M. Kaufmann

Alan H. Kawamoto

David Kellen

Colleen Kelley

Ferenc Kemény

Vera Kempe

Yoed Nissan Kenett

Christopher Kent

Gideon Keren

Alan W. Kersten

Yoav Kessler

Menusch Khadjavi

Melissa Kibbe

Stefan Kiebel

Andrea Kiesel

Alan Kingstone

John Kirby

Anastasia Kiyonaga

Maria Klatte

Joshua Klayman

Max Kleiman-Weiner

Thomas Kleinsorge

Lauren Knott

Victoria Knowland

Barbara J. Knowlton

Annemarie Kocab

Dirk Koester

Kami Koldewyn

Jonathan Kominsky

Talia Konkle

Agnieszka Konopka

Elizaveta Konovalova

Wouter Kool

Jonathan Koppel

Asher Koriat

Anita Körner

Wilma Koutstaal

Ian Krajbich

Ralf Krampe
Jens Kreitewolf

Horst Julius Krist

Judith F. Kroll

Stefanie Kuchinsky

Sarah Kucker

Christof Kuhbandner

Anton Kühberger

Anna Katharina Kuhlen

Beatrice Kuhlmann

Anuenue Kukona

Eugenia Kulakova

Victor Kuperman

Christopher A. Kurby

Benedek Kurdi

Kenneth J. Kurtz

Dave Kush

Peter Kvam

Bruno Laeng

Maria Sol Lago Huvelle

Catherine Laing

Daniel Lakens

Elke B. Lange

Naomi Langerock

Christine Larson

Nadine Lavan

Andrew Leber

Jason P. Leboe

Amy Lebkuecher*

Juliana K. Leding

Eun-Kyung Lee

Jonathan Lee

Jo-Anne LeFevre

Eric Legge

Minna Lehtonen

Kristin Lemhöfer

Karolina Lempert

Dan Levin

Tasha Lewis

Jixing Li

Xingshan Li

Melissa Libertus

Baptist Liefooghe

Lindsey Lilienthal

Diane Lillo-Martin

Shane Lindsay

Steve Lindsay

Marcus Lindskog

Kaitlyn Litcofsky

Daniel R. Little

Evan James Livesey

Alejandro Lleras

Vanessa Loaiza

Shayne Loft

Cai Stephen Longman

Heidi Lorimor

Psyche Loui

Stella F. Lourenco

Jonathon Love

Peter F. Lovibond

Matthew W. Lowder

Shenghua Luan

Christopher Lucas

Steven J. Luck
Steven G. Luke

Juan Lupiáñez Castillo

Gary Lupyan

Roy Luria

Boris Maciejowsky

Colin M. MacLeod

Susie MacRae*

Kevin Madore*

Joseph P. Magliano

Sam Maglio

James Magnuson

Lilla Magyari

Kyle Mahowald

Steve Majerus

Tal Makovski

Barbara C. Malt

Ivan Mangiulli

Ana Marcet*

Elizabeth Margulis

Anat Maril

Elizabeth Marsh

John Everett Marsh

Andrea Eyleen Martin

Amy Masnick

Robert Mason

Heath Matheson

Katherine M. Mathis

Fabien Mathy

Erico Matsumoto

Percival Grant Matthews

Ralf Mayrhofer

Jason McCarley

Ian McDonough

Todd McElroy

Geoffrey Logan McKinley

Janet McLean

Timothy P. McNamara

Melissa Meade

Benjamin Meagher

Björn Meder

Matt Meier

Tobias Meilinger

Hugo Mercier

Janet Metcalfe

Florent Meyniel

Marta Mielicki*

Evelyn Arko Milburn

Paul Miller

Bruce Milliken

Caitlin Mills

Fraser Milton

Julia Minson

Nicole Mirea

Jelena Mirkovic

Karen J. Mitchell

Sanako Mitsugi

Wenke Mohring

Shota Momma

Padraic Monaghan

Rani Moran

Laura Morett

Candice C. Morey

Emily Morgan
Bradley Morris

Joanna Morris

Joshua Morris

Betty Mousikou

Paul Muentener

Oliver Muller

Johannes Muller-Trede

Timothy Mullett

Kristi Multhaup

Margaret P. Munger

David J. Murray

Laura Mirela Muscalu

Thomas Mussweiler

Andriy Myachykov

James S. Nairne

Mariko Nakayama

Ian Neath

James Negen

John Neuhoff

Rochelle Newman

William Xiang Quan Ngiam

Bruno Nicenboim

Aiqing Nie

Alai Kirkland Staun Nielsen

Mark Nieuwenstein

Sharon Noh

Timothy Nokes-Malach

Dennis Norris

Robert Nosofsky

Jared Novick

Nazbanou Nozari

Mike Oaksford

Daniel Oberfeld

Natalie Ann Obrecht

Darko Odic

Carina Oehrn

Yasmina Okan

Chris Olivers

Henri Olkoniemi

Henrik Olsson

Daniel M. Oppenheimer

Kathleen Oppenheimer"

Marta Ortega-Llebaria

Padraig (Pat) G. O'Seaghdha

Adam Frederick Osth

Henry Otgaar

Acacia Overoye*

Albertyna Paciorek

Michael Page

Steven C. Pan

Yun Pan

Megan Papesh

Dan Parker

Colleen M. Parks

Philip Pärnamets

Paula Parpart

Benjamin A. Parris

Bernhard Pastötter

Kevin Paterson

Nikole Patson

Chotiga Pattamadilok 


\author{
Brennan Payne \\ Diane Pecher \\ Marius Peelen \\ David Peeters \\ Santiago Pelegrina \\ Manuel Perea \\ Francesca Peressotti \\ Lynn Perry \\ Yoni Pertzov \\ Jacques Pesnot-Lerousseau* \\ Daniel Peterson \\ Mathew Peterson \\ Jonathan Pettibone \\ Penny M. Pexman \\ Christina Pfeuffer \\ Roland Pfister \\ Gerit Pfuhl \\ Andrea M. Philipp \\ Colin Phillips \\ Steven Piantadosi \\ Laurence Picard \\ Erin Picou \\ Stefania Pighin* \\ Mark Pitt \\ Gaën Plancher \\ Frans Plank \\ Marie Poirier \\ Danielle Polage \\ Stephen Politzer-Ahles \\ Sean M. Polyn \\ Vencislav Popov \\ Christian H. Poth ${ }^{*}$ \\ Rosalind Potts \\ Wim T. J. L. Pouw \\ Paul C. Price \\ Konstantinos Priftis \\ Anat Prior \\ Robert W. Proctor \\ Sebastian Puschmann \\ Aryn Alexandra Pyke
}

Zhenghan Qi

Xiaomei Qiao

Philip T. Quinlan

Hugh Rabagliati

Ralph Radach

Rosanne Rademaker

Gabriel A. Radvansky

Jason Rajsic

Jason Gilbert Randall

David N. Rapp

Rachel Ratz-Lubashevsky*

John Rauthmann

Rolf Reber

Felix Rebitschek

Melissa Redford

Thomas S. Redick

A. David Redish

Erik D. Reichle

Jamie Reilly

Stian Reimers

Martina Riberto

Caitlin Rice
Patrick R. Rich

Lindsey Richland

Lauren L. Richmond

Timothy J. Ricker

Jörg Rieskamp

Ulrike Rimmele

Eric J. Rindal

Jacob Christian Rittich

Maria Robinson

Matthew Robison

Claudia Roebers

Jan Philipp Röer

Timo Roettger

Hannah Rohde

Doug Rohrer

Martin Rolfs

Steven Roodenrys

Benjamin Margolin Rottman

Christopher Rowland

Orly Rubinsten

Mary Rudner

Jan Rummel

Rachel Ryskin

Laura Sabourin

Jasmin Sadat

Jean Saint-Aubin

Anthony William Sali

Arthur G. Samuel

Jesse Sargent

Melanie Sauerland

Michele Scaltritti

Daniel L. Schacter

James R. Schmidt

Sebastian Schneegans

Darryl Schneider

Werner Schneider

Wolfgang Schnotz

Daniele Schön

Lael Schooler

Sascha Schroeder

Mark Schurgin

Bennett L. Schwartz

Norbert Schwarz

Rose M. Scott

Michael K. Scullin

Kendra Seaman

Carol Segar

Paul Seli

Michael J. Serra

Farzin Shamloo*

Jill Talley Shelton

Zhuanghua Shi

Yee Lee Shing

Alexander Siegel*

Robert Siegler

Jessica Siler

Nina Simms*

Murray Singer

Henrik Singmann

Miroslav Sirota

Anna Siyanova-Chanturia

Timothy J. Slattery

L. Robert Slevc
Steven Sloman

Scott D. Slotnick

Eleonore H. M. Smalle

Jonathan Smallwood

Amy Smith

Daniel Smith

Steven M. Smith

Jacqueline Snow

Nick Soderstrom

Elena Solesio-Jofre

Jack Soll

Alireza Soltani

Mojtaba Soltanlou

David Somers

Tobias Sommer

Lisa K. Son

Jeronimo Cassol Soro

David Soto

Alessandra S. Souza

Julia Spaniol

Pietro Spataro

Laura Jane Speed

Maarten Speekenbrink

Mikhail S. Spektor

Charles Spence

Giacomo Spinelli

Simone A. Sprenger

Mahesh Srinivasan

Dominic Standage

David Stawarczyk

Zsofia Stefan*

Karsten Steinhauer

Andrew Stewart

Neil Stewart

Mark Steyvers

Justin Storbeck

Chris N. H. Street

Helge Strøms $\varnothing$

Patrick Sturt

Jihyun Suh*

David William Sutterer

Tamara Swaab

Benjamin Swets

Briony Swire Thompson

Emily Szkudlarek

Karl Szpunar

Marcus Taft

Melanie Takarangi

Eyal Tal

Jennifer Talarico

Debbie Talmi

Joyce Tam*

Uma Tauber

Joanne S. H. Taylor

Robert Taylor

Gerry Tehan

Eylul Tekin*

Katya Tentori

Jan Theeuwes

Keith W. Thiede

Clarissa Thompson

Dorthe Thomsen

Christopher Thortenson
Anna Thorwart

Malathi Thothathiri

Renee Timmers

Debra Titone

Shingo Tokimoto

Natasha Tokowicz

Ivan Tomic*

Ilan Torgovitsky*

Joe Toscano

Greg Trafton

Matthew J. Traxler

Patrick C. Trettenbrein*

Konstantinos Tsetsos

Jonathan Tullis

Alexandra Turcan

Agnieszka Tymula

Joseph Tzelgov

Taiji Ueno

Tomer Ullman

Monika Undorf

Oyku Uner*

Christoph Ungemach

Christian Unkelbach

Igor S. Utochkin

Tugba Uzer

François Vachon

Miguel A. Vadillo

Jorge Valdes Kroff

Ronald van den Berg

Stefan Van der Stigchel

Kristin Van Engen

Tamara van Gog

Stephen Charles Van Hedger

Janet G. Van Hell

Dirk van Mooselaar

Henk van Steenbergen

Marieke van Vugt

Joshua VanArsdall

André Vandierendonck

Kristy vanMarle

Sashank Varma

Martin R. Vasilev

Awel Vaughan-Evans

Kalif Vaughn

Aaron Veldre

Michael F. Verde

Marta Vergara-Martinez

Evie Vergauwe

Paul Verhaeghen

Bram Vervliet

Willem Verwey

Timothy Vickery

Malte Viebahn

Pablo Leon Villagra*

David P. Vinson

Renee Visser

Claudia Christina von Bastian

Titus von der Malsburg

Bettina von Helversen

Adrian von Mühlenen

Jacqueline von Seth*

Momme von Sydow 
Chris Wahlheim

Lukasz Walasek

Peter Walker

Jeffrey D. Wammes

Hongbin Wang

Andrew Ward

Emily Ward

Geoff Ward

Richard Ward*

Jill Anna Warker

Andrea Weber

Kristin Weingartner

Patrick Weis

Steven Marc Weisberg

Daniel Weiss

Rebecca Weldon

Stephanie Wells*

Matthew B. Welsh

Yun Wen
Gill West

Chris Westbury

Katherine White

Corey White

David White

Katherine White

Peter A. White

Sarah J. White

David Whitebread

Peter S. Whitehead

Carol Whitney

Nicole Wicha

Iris Wiegand

Alex Wiegmann

Seth Wiener

Eva Wiese

Carrick C. Williams

Ulrike Willinger

Andy J. Wills
Brent Wilson

Jeffrey Witzel

John T. Wixted

Darrell A. Worthy

Fuyun Wu

Peter Wuehr

Dirk U. Wulff

Jimmy Xia*

Ming Xiang

Jeremy Yamashiro

Ming Yan

Veronica Yan

Chin-Lung Yang

Jinmian Yang

Melvin J. Yap

Nick Yeung

Hyungwook Yim

Si On Yoon
Galit Yovel

Chen $\mathrm{Yu}$

Christine S. P. Yu*

Lili Yu

Carole Yue

Nadira Yusif*

Alexandria Zakrzewski

Jonas Zaman*

Chuanli Zang

Dasa Zeithamova

Jiaxiang Zhang

Mintao Zhao

Yi Zheng

Yangzi Zhou*

Johannes C. Ziegler

Michael K. Ziessler

Megan Zirnstein

Phillip Zoladz

* Denotes a reviewer who co-reviewed under the supervision of a primary reviewer. 